\section{Effect of Rauwolscine on the Action of Adrenaline on Blood Pressure}

RAUWOLSCINE $\left(\mathrm{C}_{21} \mathrm{H}_{26} \mathrm{O}_{3} \mathrm{~N}_{2}\right)$, the active principle of Rauwolfia canescens, has been isolated by Chatterjee, and its chemical nature has been studied ${ }^{1,2}$. The pharmacology of this alkaloid has recently been investigated by Mukherjee and $\mathrm{Sen}^{3,4}$. This compound has been found to be hypotensive. I have observed that the intravenous injection of $1 \mathrm{mgm}$. per $\mathrm{kgm}$. of body weight of rauwolscine completely annuls the action of adrenaline on blood pressure in cats when injected intravenously in 1 c.c. doses of $1: 100,000, \quad 1: 50,000, \quad 1: 20,000$ and $1: 10,000$ strengths of adrenaline. This antagonistic action of rauwolscine to adrenaline has also been studied on amphibian and mammalian isolated heart muscles by perfusion technique. The normal amphibian and mammalian hearts are always stopped when perfused with 1 c.c. of $1: 1,000$ of adrenaline (P.D.). These hearts cannot be revived. 1 c.c. of $1: 20,000$ dilution of rauwolscine can, however, check this toxic action of adrenaline on the heart. Rauwolscine cannot counteract the glycogenolytic action of adrenaline. The mechanism of action of rauwolscine against adrenaline is now being investigated in detail.

I am indebted to Prof. A. Chatterjee, professor of chemistry, Lady Brabourne College, for kindly supplying the rauwolscine for this experiment.

\section{J. N. MuKherJeE}

Department of Physjology,

University College of Science, Calcutta 9.

Sept. 7.

${ }^{1}$ Chatterjee (né, Mukherjee), A., J. Ind. Chem. Soc., 18, 33 (1941); $18,485(1941) ; 20,11(1943) ; 23,6(1946) ; 28,29(1951)$ ${ }^{2}$ Chatterjee (ne Mukherjee), A., Science and Culture, 8, 40 (1942). ${ }^{3}$ Mukherjee, J. N., Science and Culture, 18, 338 (1953).

- Mukherjee, J. N., and Sen, P. B., Ind. J. Physiol. and Allied Sci. 7, 57 (1953); $7,109(1953) ; \gamma, 148$ (1953).

\section{A New Type of Autonomic Blocking Agent}

THE useful autonomic ganglionic blocking agent hexamethonium, and some of its closely related derivatives, possess quaternary ammonium nitrogen atoms. The activity of the compounds of this type has commonly been associated with the quaternary ammonium salt structure. Certain side-effects of drugs of this type may also be attributed to their quaternary salt nature. - In exploring a series of compounds related to W.R.L. No. 51-143, 1-methyl3 -( $\delta$-dimethylaminobutyl)piperidine dihydrochloride, it was discovered that powerful ganglionic blocking activity is also possessed by tertiary amines. 'The importance of this discovery lies in the facts that not only are these tertiary amines at least as potent and much less toxic than hexamethonium derivatives, but also, lacking the quaternary salt groups of the latter compounds, they should be free of many of the aforementioned side-effects.

On intravenous injection in anæesthetized cats and dogs, W.R.L. No. 51-143 blocks the autonomic ganglia and causes falls in blood pressure comparable to those obtained with hexamethonium chloride. Injected epinephrine is not blocked. On oral administration, equimolar doses of W.R.L. No. 51-143 last longer than hexamethonium in lowering the blood pressure.

Other tertiary amines and quaternary ammonium salts of the same series are also being investigated.
Detailed pharmacological and chemical reports on this group of substances will be made elsewhere.

Extensive work is in progress to exploit these findings by the examination of numerous other related series of compounds of quite varied structures.

Stata Norton

Arthur P. Phillits

Wellcome Research Laboratories,

Tuckahoe 7, New York. July 21.

\section{Urinary Excretion of Acid and Neutral Deoxyribonuclease by Rats under Normal and Post-Irradiation Conditions}

INTEREST in devising methods of studying tissue breakdown after irradiation has led us to investigate the deoxyribonucleases in body fluids on the premise that these enzymes are liberated only by damaged or destroyed cells. We have reported previously on the increased urinary excretion of the neutral deoxyribonuclease by rats exposed to lethal doses of $\mathrm{X}$-rays ${ }^{1}$. The purpose of the present communication is to describe a similar but more prompt increase in the urinary excretion of an acid deoxyribonuclease $(p \mathrm{H}$ optimum 5.6) by irradiated rats. This is the enzyme which has been studied recently in extenso by Allfrey and Mirsky ${ }^{2}$. It has been found in many tissues, especially those capable of active proliferation. The urinary acid deoxyribonuclease is partially inhibited by magnesium ion and citrate $(0 \cdot 1 M)$; its activity is not affected by antimony sulphate, sodium arsenate and extract of the pigeon crop gland, all of which inhibit the neutral deoxyribonuclease $e^{3,4}$.

In our experiments, twelve-hour samples of urine were collected from the experimental rats before and after total-body exposure to 700 roentgens of $250 \mathrm{kV}$. $\mathrm{X}$-rays ${ }^{1}$. The urine was diluted with an equal volume of a $1: 100(\mathrm{v} / \mathrm{v})$ aqueous solution of the chelating agent, versene, in order to remove magnesium ions. One ml. of the diluted urine was buffered with $1.5 \mathrm{ml}$. of a $0 \cdot 2 M$ acetate buffer $(p H \mathbf{H} \cdot 6)$, and the solution was incubated with $0.5 \mathrm{ml}$. of a highly polymerized solution of sodium deoxyribonucleate prepared according to the method of Kay and Dounce ${ }^{5}$. The enzymatic reaction was stopped after three hours of incubation at $37^{\circ} \mathrm{C}$. by addition of $0.8 \mathrm{ml}$. of $3.6 \mathrm{M}$ trichloracetic acid, and the deoxypentose phosphorus was determined in the supernatant fluid of the centrifuged solution. The following data demonstrate the marked increase in the deoxyribonuclease activity after irradiation of 10 rats :

\begin{tabular}{|l|r|r|}
\hline \multicolumn{2}{|c|}{ DeoxyribontClease Activitr } \\
\hline & Range & Average \\
\hline $\begin{array}{l}\text { Before irradiation } \\
\text { (12 hr., 1 day pre-exposure) }\end{array}$ & $3 \cdot 50-7 \cdot 03$ & $4 \cdot 92$ \\
$\begin{array}{l}\text { After irradiation } \\
\text { (6-18 hr. post-exposure) }\end{array}$ & $15 \cdot 96-33 \cdot 96$ & $28 \cdot 20$ \\
\hline
\end{tabular}

The deoxyribonuclease activity is calculated for the twelve-hour collection period and is expressed as acidsoluble deoxypentose-phosphorus released per hour of incubation at $37^{\circ} \mathrm{C}$.

Our results leave no doubt that there are two deoxyribonucleases in rat urine, and that the activities of both enzymes increase after total-body exposure of the animals to X-rays. These findings strongly suggest that the increased activity, particularly of the acid enzyme, is related to the destruction of radiosensitive cells. 